\title{
Perfil epidemiológico dos pacientes atendidos na clínica da dor do Centro Universitário de João Pessoa- Unipê
}

\author{
Epidemiological profile of patients attended at the pain clinic at the Centro Universitário de João
}

Pessoa - Unipê

Perfil epidemiológico de los pacientes atendidos en la clínica del dolor del Centro Universitário de

João Pessoa - Unipê

Lisandra Maria Batista Galdino ORCID: https://orcid.org/0000-0003-0041-7845

Centro Universitário de João Pessoa, Brasil

E-mail: galdinolisandra@gmail.com

Thauany Vasconcelos Soares da Silva ORCID: https://orcid.org/0000-0002-6831-9224

Universidade Federal da Paraíba, Brasil

E-mail: vasconcelosthauany@gmail.com

Herrison Félix Valeriano da Silva

ORCID: https://orcid.org/0000-0001-6714-3151 Universidade Federal da Paraíba, Brasil E-mail: herrison.felix.vds@gmail.com

Vanessa Milena Rocha Silva Soares ORCID: https://orcid.org/0000-0001-9077-0129

Centro Universitário de João Pessoa, Brasil E-mail: Vanessamilenna2@gmail.com

Livian Isabel de Medeiros Carvalho ORCID: https://orcid.org/0000-0001-7605-1523 Faculdade Nova Esperança, Brasil E-mail: isabel.livian@hotmail.com Danilo de Moraes Castanha ORCID: https://orcid.org/0000-0002-9199-8018 Universidade Federal da Paraíba, Brasil E-mail: danilo.castanha@hotmail.com

Rachel Christina de Queiroz Pinheiro

ORCID: https://orcid.org/0000-0002-8044-7544 Centro Universitário de João Pessoa, Brasil E-mail: odontogrupo@gmail.com

\begin{abstract}
Resumo
Esta pesquisa foi um estudo descritivo e quantitativo, que teve como finalidade avaliar o perfil dos pacientes atendidos na Clínica da dor em uma instituição privada. Para tanto, foram atendidos os objetivos de caracterizar os pacientes quanto ao sexo, faixa etária e grau de instrução e verificar prevalência de sinais e sintomas dos casos de DTM e Dor Orofacial. A pesquisa foi desenvolvida nas dependências da Clínica Escola de Odontologia do Centro Universitário de João Pessoa - Unipê. A amostra foi obtida de forma conveniente, constituída por 51 prontuários. Todas as informações necessárias foram registradas em um formulário online para posterior tratamento estatístico dos dados com auxílio de ferramentas estatísticas descritivas. 84,31\% eram pacientes do sexo feminino e 15,69\% eram do sexo masculino. A faixa etária mais recorrente foi a de 30 a 40 anos representando $31,37 \%$ dos participantes, em seguida 20 a 30 anos com 23,53\%. A dor associada a outro sintoma, constitui 52,94\% dos motivos pelo qual os pacientes têm procurado atendimento, 59\% dos pacientes foram diagnosticados com DTM articular e DTM muscular, $72 \%$ apresentaram bruxismo do sono e em vigília simultaneamente, $92 \%$ diagnosticados com deslocamento de disco com redução. Os músculos mais acometidos foram o corpo do masseter superficial (51\%), seguido do músculo temporal anterior $(41,2 \%)$. Concluiu-se que as mulheres entre 20 e 40 anos têm procurado o serviço com queixa de dor associada a outros sintomas, sendo os diagnósticos de DTM muscular e articular e a associação dos dois tipos de bruxismo os mais recorrentes no serviço.
\end{abstract}

Palavras-chave: Epidemiologia; Articulação temporomandibular; Músculos mastigatórios. 


\begin{abstract}
This research was a descriptive and quantitative study, which aimed to evaluate the profile of patients seen at the Pain Clinic in a private institution. To this end, the objectives of characterizing patients regarding gender, age group and education level and verifying the prevalence of signs and symptoms of TMD and Orofacial Pain cases were met. The research was carried out at the Clínica Escola de Odontologia of Centro Universitário de João Pessoa - Unipê. The sample was conveniently obtained, consisting of 51 medical records. All necessary information was recorded in an online form for later statistical treatment of the data with the aid of descriptive statistical tools. $84.31 \%$ were female patients and $15.69 \%$ were male. The most recurrent age group was 30 to 40 years old, representing $31.37 \%$ of the participants, followed by 20 to 30 years old with $23.53 \%$. Pain associated with another symptom constitutes $52.94 \%$ of the reasons why patients have sought care, 59\% of patients were diagnosed with joint TMD and muscle TMD, 72\% had sleep bruxism and while awake simultaneously, 92\% diagnosed with disk displacement with reduction. The most affected muscles were the body of the superficial masseter $(51 \%)$, followed by the anterior temporalis muscle $(41.2 \%)$. It was concluded that women between 20 and 40 years old have sought the service with complaints of pain associated with other symptoms, with diagnoses of muscular and joint TMD and the association of the two types of bruxism being the most recurrent in the service.
\end{abstract}

Keywords: Epidemiology; Temporomandibular joint; Masticatory muscles.

\title{
Resumen
}

Esta investigación fue un estudio descriptivo y cuantitativo, que tuvo como objetivo evaluar el perfil de los pacientes atendidos en la Clínica del Dolor en una institución privada. Para ello, se cumplieron los objetivos de caracterizar a los pacientes en cuanto al sexo, grupo de edad y nivel educativo y verificar la prevalencia de signos y síntomas de los casos de TTM y Dolor Orofacial. La investigación se llevó a cabo en la Clínica Escola de Odontologia del Centro Universitário de João Pessoa - Unipê. La muestra se obtuvo convenientemente, compuesta por 51 historias clínicas. Toda la información necesaria se registró en un formulario en línea para su posterior tratamiento estadístico de los datos con la ayuda de herramientas estadísticas descriptivas. El 84,31\% eran mujeres y el 15,69\% hombres. El grupo de edad más recurrente fue el de 30 a 40 años, que representó el 31,37\% de los participantes, seguido por el de 20 a 30 años con un 23,53\%. El dolor asociado a otro síntoma constituye el 52,94\% de los motivos por los que los pacientes han buscado atención, el 59\% de los pacientes fueron diagnosticados de DTM articular y DTM muscular, el 72\% tenía bruxismo del sueño y estando despiertos simultáneamente, el 92\% diagnosticaron desplazamiento discal con reducción. Los músculos más afectados fueron el cuerpo del masetero superficial (51\%), seguido del músculo temporal anterior $(41,2 \%)$. Se concluyó que mujeres entre 20 y 40 años han acudido al servicio con quejas de dolor asociado a otros síntomas, siendo los diagnósticos de TTM muscular y articular y la asociación de los dos tipos de bruxismo los más recurrentes en el servicio.

Palabras clave: Epidemiología; Articulación temporomandibular; Músculos masticatorios.

\section{Introdução}

O distúrbio da articulação temporomandibular (DTM) é uma condição complexa que engloba uma série de sintomas clínicos que se caracterizam principalmente por dores na região temporomandibular ou músculos mastigatórios, limitações na função da mandíbula e som da ATM durante o movimento mandibular (Özsinc et al. 2020).

Para Leeuw e Klasser (2018), dor é o sintoma mais frequentemente relatado, podendo ser localizada nos músculos mastigatórios e/ou região pré-auricular, sendo agravada pela mastigação ou outra atividade mandibular. Outros sintomas como limitação ou assimetria dos movimentos, ruídos na ATM, aumento da tensão dos músculos mastigatórios, fadiga muscular, dores de cabeça e desgaste oclusal associado a parafunções como, por exemplo, bruxismo e aperto dentário, também podem ser associados a DTM.

A dor orofacial pode influenciar a qualidade de vida mais do que outras condições sistêmicas, como a diabetes, hipertensão ou úlcera. Os indivíduos que apresentam tal condição vivenciam grandes mudanças no seu dia a dia, tais como: perda de dia de trabalho, ausência de relacionamento com familiares e amigos, insatisfação com a condição bucal, ingestão de medicamento e modificação na dieta alimentar (Kuroiwa et al., 2011).

Para Okerson (2008), o caráter multifatorial da etiologia das DTM permite que inúmeros fatores etiológicos contribuam para o aparecimento dos sinais e sintomas relacionados ao sistema estomatognático, oriundas da alteração da função natural deste sistema. Fatores sistêmicos são capazes de diminuir a tolerância fisiológica do indivíduo a uma lesão ou à dor. Como exemplo, podem ser citadas as interferências emocionais e afetivas na leitura da dor e da disfunção, baixo 
condicionamento físico, má qualidade nutricional e do sono. Dentre os fatores locais estão a interferência oclusal, o traumatismo muscular ou articular e os hábitos parafuncionais.

Medeiros et al. (2011) afirmam que os hábitos parafuncionais são comuns, e vem sendo considerados causas possíveis de DTM. As parafunções, comparadas com os comportamentos funcionais, como mastigação, deglutição e fala, parecem não ter finalidade funcional. Tais hábitos deletérios seriam um meio de liberação inconsciente de tensões emocionais. As parafunções geralmente não acarretam prejuízos ao sistema estomatognático. Entretanto, quando tal atividade excede a tolerância fisiológica do indivíduo, ela pode causar prejuízos à dentição, à musculatura ou à ATM.

Wahlund (2003) analisou estudos de prevalência que demonstraram que sintomas da DTM aumentam e diminuem com a idade, de modo que a gravidade é registrada na meia-idade. Mulheres, particularmente em terceira e quarta década são o gênero e faixa etária mais acometidos pelos sintomas de disfunção temporomandibular.

Iodice (2019) afirmou que a prevalência de subtipos de DTM vem sendo amplamente investigadas em populações de pacientes, relatando que aproximadamente $10 \%$ dos adultos são afetados pela dor na DTM, levando a considerá-la como a condição de dor orofacial mais comum. Afirmou também que uma quantidade menor de estudos avaliou este fato como problema na população em geral.

Portanto, o objetivo deste estudo foi avaliar o perfil epidemiológico dos pacientes atendidos na Clínica da dor em uma instituição privada. Para tanto, foram atendidos os seguintes objetivos específicos: caracterizar os pacientes quanto ao sexo, faixa etária e grau de instrução; verificar prevalência de sinais e sintomas dos casos de DTM e Dor Orofacial descritos nos prontuários analisados.

\section{Metodologia}

Tratou-se de um estudo descritivo e quantitativo, que teve como finalidade avaliar o perfil dos pacientes atendidos na Clínica da dor de uma instituição privada. Segundo Silva e Muszkat (2005), a pesquisa descritiva tem como objetivo apontar características de uma certa população ou fato, além do estabelecimento das relações entre as variáveis. Já a pesquisa com caráter quantitativo é por eles definida como tudo que pode ser quantificável, ou seja, ser traduzido em números as opiniões e informações, a fim de classificá-las e analisá-las.

A pesquisa foi desenvolvida nas dependências da Clínica Escola de Odontologia do Centro Universitário de João Pessoa - Unipê, situado na Br 230 - Km 22 S/N - Campus do UNIPÊ - 58053-000, Joao Pessoa - PB.

O universo desta pesquisa foi constituído, de forma conveniente, por todos os prontuários de pacientes atendidos na Clínica da Dor, da Clínica Escola de Odontologia do Centro Universitário de João Pessoa - Unipê, sendo um total de 118 pacientes.

Para formar a amostra, foram incluídos na pesquisa os prontuários que possuíram ficha de anamnese de DTM e Dores Orofaciais anexada ao prontuário, e que foram atendidos entre os semestres de 2018.1 e 2020.1, assim como aqueles que dispunham da ficha de anamnese com todos os campos de interesse da pesquisa devidamente preenchidos. Os prontuários que não possuíam ficha de anamnese preenchida na Clínica Da Dor da Clínica Escola de Odontologia do Centro Universitário de João Pessoa - Unipê, e pacientes menores de 18 anos foram excluídos da amostra.

Como instrumento para a coleta de dados desta pesquisa, foi utilizado um formulário (Apêndice A), elaborado pelo aluno pesquisador e pelo orientador, de acordo com os objetivos da pesquisa, onde foram colocadas as informações a serem coletadas das fichas de anamnese de DTM e Dores Orofaciais. 
Os dados coletados referentes as características gerais dos pacientes foram: sexo, faixa etária e grau de instrução. Para os dados referentes as impressões diagnósticas iniciais, foram coletados os dados referentes à: queixa principal; presença de DTM; tipo de DTM; músculos acometidos pela DTM muscular.

Com base nestes dados, foram a preenchidos 51 formulários com os prontuários que atenderam aos critérios delimitados por esta pesquisa, formando assim a amostra.

A pesquisa respeitou os aspectos éticos da Resolução 466/2012, que descreve as normas de pesquisa com seres humanos, tendo a coleta dos dados iniciada somente após a aprovação do Comitê de Ética em Pesquisa do Centro Universitário de João Pessoa-PB sob o número 4.129.053.

A coleta de dados foi realizada por uma única pesquisadora, que transferiu para um formulário online todas as informações relevantes a pesquisa, de acordo com os objetivos do estudo. Após a finalização da coleta de dados, os mesmos foram tabulados e transferidos para um banco de dados construído na ferramenta Excel do pacote Microsoft Office Professional Plus 2016, afim de facilitar o processo de análise de dados.

A análise estatística foi realizada no programa SigmaPlot 12.5 (Systat Software Inc., San Jose, CA, U.S.A). Nesta pesquisa o número de pessoas foi relativamente pequeno $(\mathrm{n}=51)$ e as variáveis avaliadas são nominais, assim não tem distribuição normal (distribuição Gaussiana) o que justifica o uso de análise não paramétrica para avaliação da associação e independência entre as variáveis.

Neste sentido, o teste exato de Fisher [intervalo de confiança de 95\%; nível de significância $\alpha=0,05(5 \%, \mathrm{P}<0,05)$ ] foi utilizado para comparar as proporções dos tipos de DTMs, bruxismos e deslocamentos de disco. Os resultados foram expressos em forma de número de pessoas e percentuais correspondentes, gerados em ambos os programas utilizados.

\section{Resultados e Discussão}

Os dados obtidos correspondentes as características gerais dos sujeitos dessa pesquisa foram dispostas na Tabela 1, onde pode-se observar que dos 51 prontuários analisados, 84,31\% eram de pacientes do sexo feminino e 15,69\% eram do sexo masculino. A faixa etária mais recorrente foi a de 30 a 40 anos (31,37\%), em seguida 20 a 30 anos (23,53\%), mais de 60 anos $(17,65 \%), 40$ a 50 anos (15,69\%), 50 a 60 anos (7,84\%) e 18 a 20 anos (3,92\%). Os dados referentes ao grau de instrução não puderam ser coletados pois o campo não estava preenchido nos prontuários.

Tabela 1 - Características gerais dos sujeitos da pesquisa.

\begin{tabular}{lll}
\hline Variável & Número de pessoas & Percentual (\%) \\
\hline Sexo & 43 & $84,31 \%$ \\
Feminino & 8 & $15,69 \%$ \\
Masculino & 51 & $100 \%$ \\
TOTAL & & \\
\hline Faixa etária & 2 & $3,92 \%$ \\
$18-20$ anos & 12 & $23,53 \%$ \\
$20-30$ anos & 16 & $31,37 \%$ \\
$30-40$ anos & 8 & $15,69 \%$ \\
$40-50$ anos & 4 & $7,84 \%$ \\
$50-60$ anos & 9 & $17,65 \%$ \\
$>60$ anos & 51 & $100 \%$ \\
TOTAL & & \\
\hline
\end{tabular}

Fonte: Dados da pesquisa. 
Estes resultados corroboram com os resultados do estudo realizado por Silveira et al. (2007) onde fora observado que dos 221 pacientes avaliados, 48 pacientes foram considerados como necessitando de tratamento para DTM (índice de DTM moderada e severa), destes 35 pertenciam ao gênero feminino (72,9\%) e 13 ao masculino $(21,1 \%)$.

Os resultados desta pesquisa são semelhantes aos achados da grande maioria dos estudos dispostos na literatura, os quais afirmam que o sexo feminino possui mais do que duas vezes o risco de desenvolver DTM. Entretanto, outros fatores de igual importância, devem ser levados em consideração, tais como: condições gerais de saúde, distúrbios de dor crônica, idade, local de estudo, etnia e fatores psicossociais e genéticos (Bueno et al., 2018).

As mulheres são apontadas como as mais afetadas por patologias ósseas e psicossociais, isto pode ser dar devido ao fato de que há maior prevalência de sinais e sintomas de DTM em idade reprodutiva. Os hormônios sexuais, principalmente o estrogênio, possuem desempenho significativo na sensibilidade dolorosa, incluindo os músculos da mastigação e na patogênese das DTM, no limiar da dor, e sua tolerância tende a variar de acordo com a fase do ciclo menstrual (Ferreira; Silva; Felício, 2016). Porém é importante relatar que há uma relação de comorbidade entre sintomas de disfunção e problemas gerais de saúde. (Basto et al., 2017).

Quanto as faixas etárias, os achados nesta pesquisa são semelhantes aos encontrados por Basto et al. (2017), que ao analisarem vários estudos perceberam que faixa etária mais acometida por problemas de DTM apresenta-se entre 20-40 anos e 35-44 anos. Entretanto, é importante ressaltar que o avanço da idade tem se mostrado como um fator associado a necessidade de tratamento da disfunção. A idade deve ser considerada nos pacientes com DTM no momento da escolha da estratégia de tratamento.

Foram analisados dados referentes a queixa principal dos pacientes atendidos pelo serviço (Tabela 2). Pode-se observar que a dor, sendo associada ou não a outro sintoma, é o motivo principal pelo qual os pacientes têm procurado atendimento $(52,94 \%)$. Todavia, a diferença percentual entre os fatores de procura por atendimento odontológico não é estatisticamente significativa $(\mathrm{P}>0,05)$. Uma explicação para isto é que o tamanho da amostra não é grande o suficiente.

Observou-se que 92,16\% dos pacientes que procuraram o serviço apresentaram diagnóstico de DTM. Quando analisados os dados referentes ao tipo de DTM (Tabela 2), 59\% dos pacientes foram diagnosticados com DTM articular e DTM muscular, resultado que diferiu estatisticamente $(\mathrm{P}<0,001)$ da quantidade de pacientes que foram diagnosticados com apenas DTM articular (19\%) ou DTM muscular (14\%).

Se tratando de bruxismo (Tabela 2), apenas 32 pacientes apresentaram tal condição. Destes, $72 \%$ apresentaram bruxismo do sono (BS) e em vigília (BV) simultaneamente, valor que diferiu estatisticamente $(\mathrm{P}<0,001)$ da quantidade de pessoas que apresentaram apenas bruxismo em vigília (22\%).

Dos 40 pacientes diagnosticados com DTM articular (Tabela 2), 38 apresentaram deslocamento de disco, sendo 92\% destes diagnosticados com deslocamento de disco com redução, percentual que diferiu estatisticamente $(\mathrm{P}<0,001)$ da quantidade de pessoas que foram diagnosticadas com deslocamento de disco sem redução (8\%). 
Tabela 2 - Características específicas dos sujeitos da pesquisa.

\begin{tabular}{|c|c|c|}
\hline Variável & Número de pessoas & Percentual (\%) \\
\hline \multicolumn{3}{|l|}{ Queixa principal } \\
\hline Dor & 18 & $35,30 \%$ \\
\hline Dor e outros sintomas & 27 & $52,94 \%$ \\
\hline Sem dor, mas com outros sintomas & 5 & $9,80 \%$ \\
\hline Não informado & 1 & $1,96 \%$ \\
\hline TOTAL & 51 & $100 \%$ \\
\hline \multicolumn{3}{|l|}{ Tipo de DTM } \\
\hline DTM articular & 10 & $19,61 \% \mathrm{~b}$ \\
\hline DTM muscular & 7 & $13,73 \%{ }^{b}$ \\
\hline Ambas as DTMs & 30 & $58,82 \% \mathrm{a}^{\mathrm{a}^{* * *}}$ \\
\hline Não apresentaram & 4 & $7,84 \%$ \\
\hline TOTA & 51 & $100 \%$ \\
\hline \multicolumn{3}{|l|}{ Bruxismo } \\
\hline Bruxismo do sono & 2 & $6 \%$ a,b \\
\hline Bruxismo em vigília & 7 & $22 \%$ \\
\hline Ambos os bruxismos & 23 & $72 \% \mathrm{a}^{* * *}$ \\
\hline TOTAL & 32 & $.63 \%$ \\
\hline \multicolumn{3}{|l|}{ Deslocamento de Disco } \\
\hline Deslocamento de disco com redução & 35 & $92 \% \mathrm{a}^{* * *}$ \\
\hline Deslocamento de Disco sem redução & 3 & $7 \%^{\mathrm{b}}$ \\
\hline TOTAL & 38 & $95 \%$ \\
\hline
\end{tabular}

Diferentes letras minúsculas subscritas na mesma coluna significa diferença estatística entre os parâmetros avaliados de acordo com o teste exato de Fisher, $* * * \mathrm{P}<0,001$.

Fonte: Dados da pesquisa.

Um grande estudo transversal de base populacional (189.977 pessoas, sendo 52\% mulheres e $48 \%$ homens) realizado nos Estados Unidos apontou que 83\% dos indivíduos que sofrem de dor relacionada a DTM relataram uma condição de dor comórbida e 59\% relataram em pelo menos duas condições. A comorbidade mais relatada em pessoas com DTM foram dor, dor no pescoço e dor nas costas (Plesh; Adams; Gansky, 2011). Vários estudos têm associado a presença de cefaleia associada as sintomatologias dolorosas da DTM (List; Jensen, 2017). Um estudo realizado por Anderson et al. (2011) concluiu que adultos que frequentemente sofriam com dor de cabeça eram mais suscetíveis a manifestações de agravamento de sinais e sintomas de DTM do que indivíduos com menor frequência de cefaleia.

Um estudo realizado no Brasil por Machado et al. (2012) com 357 pacientes sintomáticos em uma clínica privada observou que os diagnósticos mais comuns foram DTM muscular com dor localizada no momento da mastigação $(\mathrm{n}=125)$ e DTM articular do tipo Deslocamento de Disco Sem Redução $(n=104)$. Outro estudo realizado na Índia com 450 estudantes de graduação e pós graduação observou que a DTM mais prevalente foi deslocamento de disco (22,6\%), seguido por síndrome da disfunção da dor miofascial com deslocamento de disco $(13,5 \%)$ e síndrome da disfunção da dor miofascial $(3,8 \%)($ Ahuja et al., 2018). 
Dor muscular mastigatória tem sido reconhecida como a queixa mais recorrente em pacientes na prática geral, sob a hipótese de que os pacientes se apresentem para tratamento com estágio crônico. Tal afirmação pode ser explicada devido ao fato de que muitos músculos ou distúrbios articulares não passam apenas despercebidos pelos pacientes e profissionais na prática geral, mas também porque a maioria dos profissionais não sabem seguir adequadamente com as condutas de tratamento (Machado et al., 2012).

Quando realizado um levantamento epidemiológico na população holandesa (total de 1.209 indivíduos de diferentes idades) pôde-se observar que 5\% da população total apresentou bruxismo em vigília e 16,5\% apresentaram bruxismo do sono, e foi concluído que o bruxismo do sono é uma condição comum na população adulta holandesa, enquanto o bruxismo na vigília é mais raro (Wetselaar et al., 2019). Já o estudo realizado com 4.403 estudantes de graduação finlandeses mostrou que o bruxismo do sono foi relatado por $21 \%$ das mulheres e $12,5 \%$ dos homens, o bruxismo da vigília por 2,0\% das mulheres e por 2,8\% dos homens, e o bruxismo do sono e da vigília por 7,2\% das mulheres e por 3,2\% dos homens (Huhtela et al., 2016). Tais resultados diferem dos resultados obtidos nesta pesquisa, isto pode ter se dado devido a quantidade relativamente menor de pessoas analisadas neste estudo.

Um estudo retrospectivo longitudinal avaliou 217 articulações em 165 pacientes (130 mulheres e 35 homens), cujas idades variaram de 11 a 64 anos na consulta inicial. No exame de ressonância magnética inicial, foram encontrados deslocamento de disco com redução em 86 articulações e deslocamento de disco sem redução em 131 articulações. Os achados mostraram que o deslocamento de disco sem redução sofreu alterações morfológicas mais graves com o passar do tempo (HU; YANG; XIE, 2016). Tais resultados divergem dos achados nessa pesquisa. Vários fatores podem ter levado a essa divergência de resultados, como a quantidade amostral ou características sociodemográficas das diferentes amostras.

Dias et al. (2016) avaliou deslocamento de disco e associou a osteoartrose. Foram avaliadas 224 imagens de ATM de pacientes com sinais e sintomas de DTM, concluindo que articulações com deslocamento anterior do disco com redução e deslocamento anterior do disco sem redução tiveram 2,73 e 8,25 vezes, respectivamente, mais probabilidade de apresentar osteoartrose.

O levantamento realizado nesta pesquisa aponta que 35 dos pacientes apresentaram sintomatologia dolorosa nos músculos avaliados durante o exame de palpação (Gráfico 1). Os músculos mais acometidos por sintomatologia dolorosa foram o corpo do masseter superficial (MS-C) (51\%), seguido do músculo temporal anterior (TA) $(41,2 \%)$ e a inserção móvel do músculo masseter superficial (MS-IM) $(39,2 \%)$. 
Gráfico 1 - Músculos acometidos por sintomatologia dolorosa.

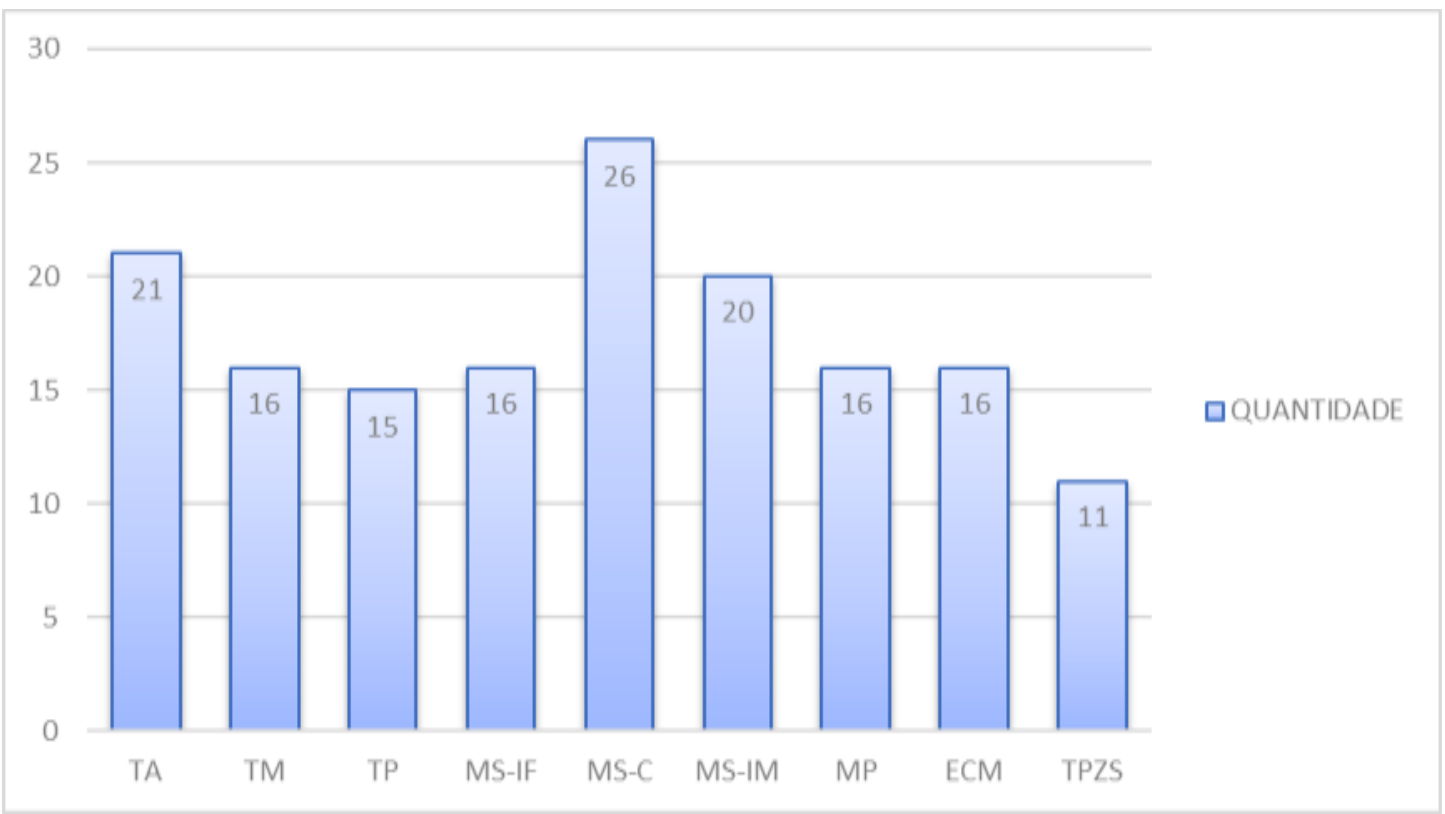

Siglas: músculo temporal anterior (TA), músculo temporal médio (TM), músculo temporal posterior (TP), músculo masseter superficial inserção fixa (MS-IF), músculo masseter superficial corpo (MS-C), músculo masseter superficial inserção móvel (MS-IM), músculo masseter profundo (MP), músculo esternocleidomastóideo (ECM), músculo trapézio superior (TPZS).

Fonte: Dados da pesquisa.

Um estudo realizado com 26 participantes avaliou a atividade muscular em vigília e durante o sono em pacientes com e sem sintomatologia dolorosa de DTM. Os achados nesse estudo mostraram que para o músculo masseter houve um maior padrão de atividade para os pacientes com sintomatologia dolorosa tanto no sono quanto em vigília. Padrões semelhantes, porém, em menor quantidade foram encontrados também no músculo temporal. Entretanto, não é possível identificar uma causa para o acontecimento, acredita-se que tenha relação com a atividade mastigatória (Khawaja et al., 2015). O estudo feito por Wrigth e North (2009) mostrou que ocorreu prevalência do músculo trapézio superior em $60 \%$ dos pacientes, o pterigóideo lateral em $50 \%$, e o masseter e feixe superficial em $47 \%$.

Já um estudo transversal realizado no Brasil, o qual buscou avaliar o limiar de dor a palpação nos músculos mastigatórios em 60 mulheres com DTM, mostrou que as participantes apresentaram diminuição do limiar de dor a palpação na área do músculo pterigoideo lateral, bilateralmente, seguido pelos músculos masseter e temporal (Herpich et al., 2018). Os resultados presentes na literatura divergem em relação a quais são os músculos mastigatórios mais acometidos por sintomatologia dolorosa, assim são necessários estudos mais aprofundados para nível de comparação.

\section{Considerações Finais}

Diante dos resultados obtidos neste estudo, tendo em vista as limitações encontradas, partindo da premissa de que existe maior prevalência de DTM que outros tipos de desordens orofaciais nos pacientes atendidos na clínica da Dor da instituição, concluiu-se que a DTM articular e a muscular associadas são as mais prevalentes no serviço. O mesmo ocorre para os pacientes que apresentam bruxismo, onde o bruxismo do sono e o em vigília foram registrados simultaneamente em uma quantidade significativamente maior de pacientes.

O perfil que foi possível traçar com os pacientes da amostra foi de mulheres, entre 20 e 40 anos, que buscaram o serviço por dor associada a outros sintomas. Há uma predominância estatisticamente significativa de deslocamento de disco com redução para os pacientes diagnosticados com DTM articular. Os músculos masseter e temporal mostraram-se como 
sendo os mais recorrentes nos exames de palpação. Isto posto, levando em consideração o reduzido fluxo de pacientes, sugerese que o estudo seja repetido no futuro, com uma amostra maior afim de comparação.

\section{Referências}

Ahuja, V., Ranjan, V., Passi, D. \& Jaiswal, R. (2018). Study of stress-induced temporomandibular disorders among dental students: An institutional study. National journal of maxillofacial surgery, 9(2), 147-154.

Anderson, G. C., John, M. T., Ohrbach, R., Nixdorf, D. R., Schiffman, E. L., Truelove, E. S. \& List, T. (2011). Influence of headache frequency on clinical signs and symptoms of TMD in subjects with temple headache and TMD pain. Pain, 152(4), 765-771.

Basto, J. M., Gonçalves, L. S., Isaías, P. H. C., Silva, R. A. D. A., Bastos, P. L. \& Figueiredo, V. M. G. (2017). Temporomandibular disorders: a literature review on epidemiology, signs and symptoms and clinical examination. Revista Da Saúde E Biotecnologia, 1(1), 66-77.

Bedran, L. M. (2019). Changes in temporomandibular joint anatomy, changes in condylar translation, and their relationship with disc displacement: magnetic resonance imaging study. Radiol Bras. 52(2), 85-91.

Bueno, C. H., Pereira, D. D., Pattussi, M. P., Grossi, P. K. \& Grossi, M. L. (2018). Gender differences in temporomandibular disorders in adult populational studies: A systematic review and meta-analysis. Journal of oral rehabilitation, 45(9), 720-729.

Carrara, S. V., Conti, P.C.R. \& Barbosa, J. S. (2010). Termo do $1^{\text {o }}$ consenso em disfunção temporomandibular e dor orofacial. Dental Press J Orthod., 15(3), 114-20.

Donnarumma, M. D. C., Muzzili, C. A., Cristiane, F. \& Nemr, K. (2010). Disfunções Temporomandibulares: Sinais, Sintomas e Abordagem Multidisciplinar. Revista CEFAC, 12(5), 788-94.

Fernandes NETO, A. J., Neves. D. F. \& Simamoto JR., P. C. (2013). Oclusão - Serie Abeno: Odontologia Essencial - Parte Clínica. Editora Artes Médicas LTDAs.

Ferreira, C. L. P., Silva, M. A. M. R. D.A \& Felício, C. M. D.E. (2016). Signs and symptoms of temporomandibular disorders in women and men. CoDAS, 28(1), 17-21.

Firmani, M., Reyes, M., Becerra, N., Flores, G., Weitzman, M. \& Espinosa, P. (2015). Bruxismo de sueño en niños y adolescentes. Rev Chil Pediatr, 86, 373-379.

Gnauck, M. et al. (2017). Knowledge and competence in temporomandibular disorders among Swedish general dental practitioners and dental hygienists. Acta Odontol Scand. 75, 429-36.

Herpich, C. M., Politti, F., Gomes, C. A. F. de P., Gloria, I. P. dos S., Amaral, A. P., Amaral, M. de F. R. de S., Herpich, G., Kalil, S. B., Gonzalez, T. de O. \& Biasotto-gonzalez, D. A. (2018). Avaliação do limiar de dor a palpação dos músculos mastigatórios em mulheres com disfunção temporomandibular de acordo com o Research Diagnostic Criteria of Temporomandibular Disorders. Revista CEFAC, 20(2), 175-181.

Hu, Y. K., Yang, C. \& Xie, Q. Y. (2016). Changes in disc status in the reducing and nonreducing anterior disc displacement of temporomandibular joint: a longitudinal retrospective study. Scientific reports, 6, 34253.

Huhtela, O. S., Näpänkangas, R., Joensuu, T., Raustia, A., Kunttu, K. \& Sipilä, K. (2016). Self-Reported Bruxism and Symptoms of Temporomandibular Disorders in Finnish University Students. Journal of oral \& facial pain and headache, 30(4), 311-317.

Iodice, G. et al. (2019). Prevalence of temporomandibular disorder pain, jaw noises and oral behaviors in an adult Italian population sample. Journal of Oral Rehabilitation, 10, 12 .

Khawaja, S. N., Mccall, W., Jr, Dunford, R., Nickel, J. C., Iwasaki, L. R., Crow, H. C. \& Gonzalez, Y. (2015). Infield masticatory muscle activity in subjects with pain-related temporomandibular disorders diagnoses. Orthodontics \& craniofacial research, 18, 137-145.

Kuroiwa, D. N., Marinell, J. G., Rampanii, M. S., Oliveira, W. \& Nicodemo, D. (2011). Temporomandibular disorders and orofacial pain: study of quality of life measured by the Medical Outcomes Study 36 - Item Short Form Health Survey. Revista Dor. 12(2).

Lal, S. J. \& Weber, K. K. (2020). Bruxism Management. In: StatPearls. StatPearls Publishing.

Leeuw R. \& Klasser G., editors. (2018). Orofacial pain: guidelines for assessment, diagnosis, and management. (6a ed.), Quintessence.

List, T. \& Jensen R. H. (2017). Temporomandibular disorders: Old ideas and new concepts. International Headache Society. $37,7$.

Medeiros, S. P., Batista, A. U. D. \& Forte, F. D. S. (2011). Prevalência de sintomas de disfunção temporomandibular e hábitos parafuncionais em estudantes universitários. RGO. Revista Gaúcha de Odontologia. 59(2).

Okeson, J. P. (2008). Tratamento das desordens temporomandibulares e oclusão. Elsevier.

Özdinç, S., PhD et al. (2020). Temporomandibular joint disorder determined by Fonseca anamnestic index and associated factors in 18- to 27-year-old university students. Cranio: the journal of craniomandibular practice, 38(5), 327-332.

Plesh O., Adams S. H. \& gansky S. A. (2011). Temporomandibular joint and muscle disorder-type pain and comorbid pains in a national US sample. J Orofac Pain. 25, 190-198. 
Research, Society and Development, v. 10, n.13, e306101321379, 2021

(CC BY 4.0) | ISSN 2525-3409 | DOI: http://dx.doi.org/10.33448/rsd-v10i13.21379

Polat, M. E. \& Yanik, S. (2019). Efficiency of arthrocentesis treatment for different temporomandibular joint disorders. International Journal of Oral and Maxillofacial Surgery. 10, 1016.

Santos, K. C. et al. (2013). Correlação entre as alterações observadas nos distúrbios internos da articulação temporomandibular avaliadas por ressonância magnética em pacientes sintomáticos. J Oral Maxillofac Surg. 71, 1504-12.

Silva, E. L. (2005). Metodologia da pesquisa e elaboração de dissertação. (4a ed.), rev. atual. UFSC.

Shah, J. P. \& Gilliams, E. A. (2008). Uncovering the biochemical milieu of myofascial trigger points using in vivo microdialysis: an application of muscle pain concepts to yofascial pain syndrome. J Bodyw Mov Ther, 12(4), 371-84

SilveiraI, A. M. et al. (2007). Prevalência de portadores de DTM em pacientes avaliados no setor de otorrinolaringologia. Rev. Bras. Otorrinolaringol. 73(4).

Tosato, J.P., Biasotto-gonzalez, D. A. \& Gonzalez, T.O. (2005). Presença de desconforto na articulação temporomandibular relacionada ao uso da chupeta. Revista Brasileira Otorrinolaringol., 71(3), 365-68.

Wahlund, K. (2003). Desordens temporomandibulares em adolescentes. Estudos epidemiológicos e metodológicos e um conjunto de estudo controlado omitido. Sueco Dent J Suppl.

Wetselaar, P., Vermaire, E. J. H., Lobbezoo, F. \& Schuller, A. A. (2019). The prevalence of awake bruxism and sleep bruxism in the Dutch adult population. Journal of oral rehabilitation, 46(7), 617-623.

Wrigth, E. F. \& North, S. L. (2009). Management and treatment of temporomandibular disorders: a clinical perspective. J Man Manip Ther. 17, $247-54$. 